\title{
Large deformation three-dimensional static analysis of deep water marine risers
}

\author{
MICHAEL M. BERNITSAS, JOHN E. KOKARAKIS and ASJHAR IMRON \\ Department of Naval Architecture and Marine Engineering, The University of Michigan, Ann Arbor, \\ MI 48109-2145, USA
}

\begin{abstract}
The problem of static three-dimensional, nonlinear, large deformation response of a marine riser is formulated within small strain theory and solved numerically. This type of analysis is necessary, for the new generation of drilling and production risers. The mathematical model takes properly into account the effects of internal and external pressure and the complete nonlinear boundary conditions, without linearizing the follower forces. The extensibility or inextensibility condition is used as the constitutive relation in the tangential direction. Torsion and bending are coupled. The external load and the boundary conditions are deformation dependent. A solution method is developed based on an incremental finite element algorithm, which involves a prediction-correction scheme. In the correction phase deformation dependent quantities are updated. The extensibility or inextensibility condition is used to reduce the degrees of freedom of the system. The numerical results of the developed computer code compare very well with available semi-analytical and numerical solutions. Three numerical applications are used to demonstrate the importance of large deformation, nonlinear and three-dimensional analyses.
\end{abstract}

Key Words: Marine risers, nonlinear, static, three-dimensional, large deformation, extensibility, torsion, deformation dependency of loads, incremental finite element

\section{INTRODUCTION}

The problem of static and dynamic behavior of marine risers has been studied extensively in the past. Initially several structurally linear models have been developed and solved using analytical, numerical or series solutions. ${ }^{6}$ In addition to nonlinear expressions for hydrodynamic drag loads it was soon realized, that nonlinear geometric and structural effects are also significant, particularly for long risers. ${ }^{1}$ Such nonlinearities are due to large deflections and slopes, three-dimensional bending, extensibility, torsion, dependency of the hydrodynamic loads on the riser deformation and nonlinear boundary conditions. The nature of these nonlinearities is explained below.

The magnitude of deflections and slopes increases with the loading parameter $\beta$ which is proportional to the lateral load, $q$, and the cube of the riser length, $L$, and inversely proportional to the bending rigidity, $E I ; \beta=q L^{3} / E I$. The corresponding structural nonlinearities become significant particularly for the new generation of drilling risers which have a length of the order of $2000 \mathrm{~m}$. Three-dimensional bending is also important for such risers because the hydrodynamic loading due to the surface waves, the ocean current, the relative motion of the riser with respect to the water and the position of the supporting offshore platform, is actually three-dimensional. Extensibility effects are important for production risers made of flexible composite material. Torsional couples arise from the variation of the relative orientation of the supporting offshore platform and the riser and the imperfections in the telescopic joint in the tensioning system and the lower ball joint, and are concen-

Accepted February 1985. Discussion closes December 1985. trated at the upper end of the riser, only a minor torsional couple is induced by the asymmetry of the relative flow due to vortex shedding. Further the hydrodynamic loads exerted on risers strongly depend on the orientation of the riser tubes with respect to the relative flow ${ }^{2,5}$ and therefore they are deformation dependent. Finally the boundary conditions are nonlinear and have two components one of which is a nonconservative (follower) force explained in Section II.

To account for these nonlinearities many nonlinear models have recently been developed. The purpose of this paper is to develop a comprehensive nonlinear small strain model for marine risers and solve it numerically. In this work, the marine riser is considered as a slender, thin walled circular beam, subject to its own weight, internal and external pressure forces due to fluids in gravity field, tension exterted at its top, distributed buoyancy from attached modules, deformation dependent hydrodynamic loads and torsional moment.

A literature review discussing the feature of the various nonlinear models published in the literature is presented in Section I. The mathematical model developed in Section II is a static, small strain, three-dimensional, large deformation model, which takes into consideration geometric and structural nonlinearities, the extensibility or inextensibility of risers and the dependency of the boundary conditions on the deformation. In Section III an incremental finite element method which implements a prediction correction scheme is used to solve the mathematical model. The accuracy of the developed computer code is shown to be very satisfactory, on the basis of results derived by numerical or series expension solutions. In Section IV several nonlinear beam problems which have been formulated and solved numerically in the past are used 
to verify the validity of the method and the accuracy of the computer code developed in this work. Finally in Section V several numerical applications on risers are used to demonstrate the importance of large deformation nonlinear and three-dimensional analyses.

\section{BACKGROUND}

Most of the riser models published in the literature use nonlinear expressions for the hydrodynamic forces. In this work the emphasis is on structural rather than hydrodynamic nonlinearities and the term 'nonlinear' used hereafter refers to the former. All nonlinear riser models which have been published in the literature, including the one developed here, are small strain models. Nordgren ${ }^{22}$ formulated and solved by finite differences the nonlinear motions of inextensible rods, and applied his model to initially vertical risers ${ }^{23}$ by linearizing the equations and neglecting torsion. His equations are valid for initially straight risers with cross sections of equal principal stiffnesses. Garrett, ${ }^{10}$ used the same equations and solved them by a finite element method increasing the degrees of freedom by introducing a continuous Lagrange multiplier which has the purpose of forcing the inextensibility condition. The additional degree of freedom accounts for the unknown tangential force.

Gnone et al. ${ }^{12}$ take into consideration a geometric nonlinearity due to the extension of riser joints but neglect, structural nonlinearities, torsion and nonlinearities in the boundary conditions. Larsen and Kavlie ${ }^{20}$ solve a twodimensional, small rotation problem, neglecting axial deformations and torsion. Fellipa and Chung ${ }^{9}$ applied the energy method to derive the equations of equilibrium and used a nonlinear expression for the longitudinal strain. Bernitsas ${ }^{3}$ has developed a nonlinear, large deformation, three-dimensional model for extensible risers. Konuk ${ }^{19}$ has solved a comprehensive nonlinear model but does not discuss important aspects of the riser problem like extensibility, deformation dependency of boundary conditions and loads. Safai $^{26}$ includes the geometric nonlinearity and his equations of bending, extension and torsion are uncoupled. Kim and Triantafyllou ${ }^{17}$ use a nonlinear expression for the longitudinal strain, while their bending equations are linear and uncoupled. Huang and Chucheepsaku1 ${ }^{14}$ have solved a nonlinear two-dimensional problem neglecting torsion and extensibility. Further, it should be mentioned that all of the above neglect the deformation dependency of the boundary conditions and particularly the one due to the follower pressure forces. Finally, even though the interactive nature of the hydrodynamic loads is usually taken into consideration, the dependency of the loads on the local orientation of the riser is neglected.

\section{MATHEMATICAL FORMULATION}

The static riser problem described in the introduction is formulated in this section assuming that the material is homogeneous, isotropic and linearly elastic, the riser elements are thin walled tubular heavy beams which are locally stiff so that plane sections remain plane after bending. Further shear deformation is neglected and the riser is assumed to be straight in the unloaded condition and having cross sections of equal principal stiffnesses. Equilibrium of forces and moments yield respectively:

$$
\mathbf{F}^{\prime}+\mathbf{q}=\mathbf{0}
$$

and

$$
\mathbf{M}^{\prime}+(\hat{t} \times \mathbf{F})+\mathbf{m}=\mathbf{0}
$$

where, $\mathbf{F}$ and $\mathbf{M}=$ internal stress resultant force and moment vectors respectively, $\mathbf{q}$ and $\mathbf{m}=$ applied force and moment per unit length, $\hat{t}=\mathrm{d} \mathbf{r} / \mathrm{d} s_{1}=$ unit local tangential vector shown in Fig. 1, the differential operator is $\mathrm{d} / \mathrm{d} s_{1}$, with respect to the arc length $s_{1}$ of the deformed centerline of the riser, bold type and " $n$ indicate vector and unit vector respectively. The constitutive relation for bending and torsion is given by Love $^{21}$ as:

$$
\mathbf{M}=B \kappa \hat{b}+H \hat{t}
$$

where $B=E I=$ bending rigidity of the cross-section, $H=$ torsional moment, $k=\left|\mathbf{r}^{\prime \prime}\right|$ is the curvature in the osculating plane, and $\hat{b}=\mathbf{r}^{\prime} \times \mathbf{r}^{\prime \prime} / \kappa=$ unit local binormal vector shown in Fig. $1 .^{8}$ Combining (1) and (2) we get the bending equation: ${ }^{17}$

$$
\begin{aligned}
& -\left(B \mathbf{r}^{\prime \prime}\right)^{\prime \prime}+\left[\left(T_{e}-B \kappa^{2}\right) \mathbf{r}^{\prime}\right]^{\prime}+\left[H\left(\mathbf{r}^{\prime} \times \mathbf{r}^{\prime \prime}\right)\right]^{\prime}+\left(\mathbf{r}^{\prime} \times \mathbf{m}\right)^{\prime}+\mathbf{q} \\
& \quad=0
\end{aligned}
$$

and the torsion equation:

$$
H^{\prime}+\hat{t} \cdot \mathbf{m}=0
$$

where, $\mathbf{r}$ is the position vector of an element on the riser centerline in the deformed state as shown in Fig. 1 given by $\mathbf{r}=x_{1} \hat{i}+x_{2} \hat{j}+\left(x_{3}+s\right) \hat{k}, T_{e}$ is the effective riser tension given by:

$$
T_{e}=T+\rho_{w g} g \frac{\pi D_{0}^{2}}{4}\left(H_{w}-z\right)-\rho_{m} g \frac{\pi D_{i}^{2}}{4}\left(H_{m}-z\right)
$$

$T=$ actual tension in the riser, $H_{w}=$ ordinate of the free surface of the water, $H_{m}=$ ordinate of the free surface of the mud, $\rho_{w}$ and $\rho_{m}$ are the densities of water and mud respectively, $D_{0}$ and $D_{i}=$ external and internal riser diameters and $z$ is measured from the lower ball joint:

$$
z=s+x_{3}
$$

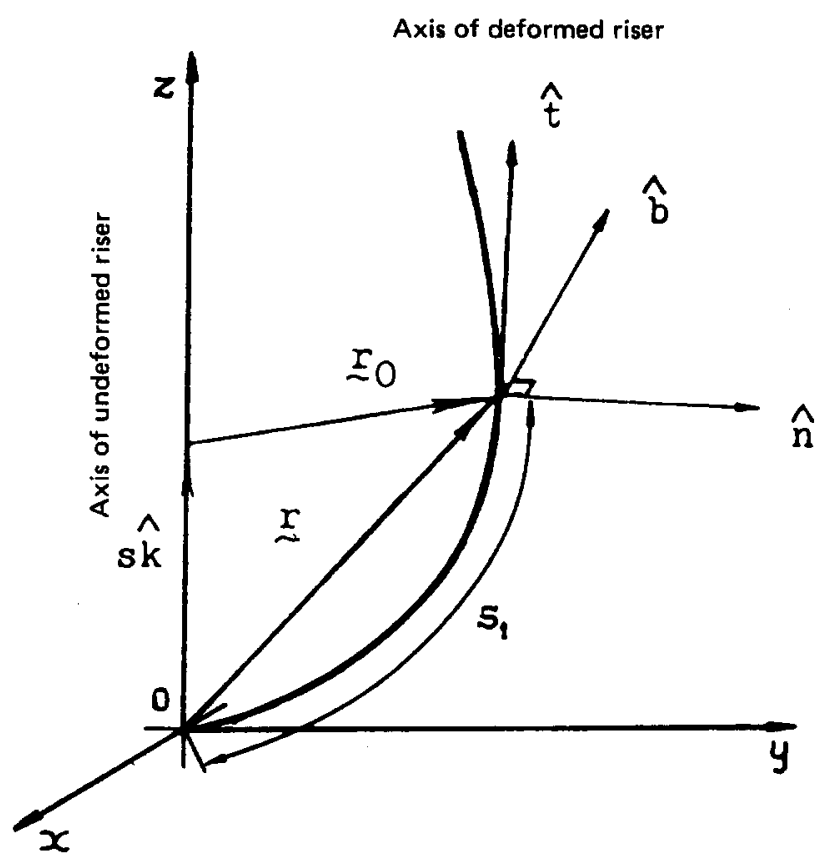

Figure 1. Position vector and principal direction for a riser point 
where $s=$ length of the riser centerline measured along $0 z$ (Fig. 1) in the initial unstrained state, and the displacement vector in the deformed state is:

$$
\mathbf{r}_{0}=\left(x_{1}, x_{2}, x_{3}\right)
$$

Further, $T_{e}$ is the tangential component of the internal force given by (9):

$$
T_{e}=\mathbf{F} \cdot \hat{t}
$$

where $\mathbf{F}$ is derived by integration of equation (1). It should be noted that the vertical component of $\mathbf{q}$ includes the effective and not the actual weight of the riser per unit length, defined as the weight of riser plus contents in water. $^{3,15}$ The actual tension $T$ also satisfies the constitutive relation:

$$
T=E A \epsilon_{t}
$$

where $E A$ is the stretching rigidity and $\epsilon_{t}$ the strain of the riser centerline in the tangential direction defined as:

$$
\epsilon_{t}=\left[\frac{\mathrm{d} \mathbf{r}}{\mathrm{d} s} \cdot \frac{\mathrm{d} \mathbf{r}}{\mathrm{d} s}\right]^{1 / 2}-1
$$

where $s$ and $s_{1}$ are related by:

$$
\frac{\mathrm{d} s_{1}}{\mathrm{~d} s}=1+\epsilon_{t}
$$

The external loads $\mathbf{q}\left(s_{1}\right)$ and external moments $\mathbf{m}\left(s_{1}\right)$ are functions of the environmental conditions and the riser orientation. Considering only the fluid drag force in Morison's formula, we have: ${ }^{2}$

$$
\mathbf{q}\left(s_{1}\right)=\left\{\begin{array}{l}
q_{1} \\
q_{2} \\
q_{3}
\end{array}\right\}=\frac{1}{2} \rho_{w} C_{d} D_{H}\left|\mathbf{V}_{n}\right|\left\{\begin{array}{l}
V_{n_{1}} \\
V_{n_{2}} \\
V_{n_{3}}
\end{array}\right\}
$$

where $C_{d}=$ drag coefficient given in refs 4 and $5, D_{H}=$ local hydrodynamic diameter and $\mathbf{V}_{n}=$ component of the relative flow velocity which is normal to the riser centerline, and can be computed as follows. Let $\mathbf{V}$ be the relative flow velocity due to waves, current and the riser motion. For the static analysis the latter is zero. If $u, v, w$ denote the components of fluid velocity due to waves and currents in the $x_{1}, x_{2}, x_{3}$ directions, then:

$$
\begin{gathered}
\mathbf{V}=u \hat{i}+v \hat{j}+w \hat{k} \\
\mathbf{V}_{n}=\hat{t} \times(\mathbf{V} \times \hat{t})=\mathbf{V}-(\hat{t} \cdot \mathbf{V}) \hat{t}
\end{gathered}
$$

and

$$
\begin{gathered}
V_{n_{1}}=u-\left[x_{1}^{\prime} u+x_{2}^{\prime} v+\left(x_{3}^{\prime}+\frac{1}{1+\epsilon_{t}}\right) w\right] x_{1} \\
V_{n_{2}}=v-\left[x_{1}^{\prime} u+x_{2}^{\prime} v+\left(x_{3}^{\prime}+\frac{1}{1+\epsilon_{t}}\right) w\right] x_{2}^{\prime} \\
V_{n_{3}}=w-\left[x_{1}^{\prime} u+x_{2}^{\prime} v+\left(x_{3}^{\prime}+\frac{1}{1+\epsilon_{t}}\right) w\right]\left(x_{3}^{\prime}+\frac{1}{1+\epsilon_{t}}\right)
\end{gathered}
$$

where $x^{\prime}=\mathrm{d} x / \mathrm{d} s_{1}$.

The couple $\mathbf{m}\left(s_{1}\right)$ arises from asymmetry in the relative flow due to vortex shedding and the presence of kill and choke lives. It is a small term compared to the torque induced by the imperfections of the tensioning system and the lower ball joint. Therefore, even though $\mathbf{m}\left(s_{1}\right)$ is included in the theoretical analysis and the computer code it is not considered in the numerical applications of Section V.

For the completion of the mathematical model specification of the following boundary conditions is needed:

(1) $\hat{t} \times \mathbf{M}$ or $\mathbf{r}^{\prime}$ at $s_{1}=0$,

(2) $\hat{t} \times \mathbf{M}$ or $\mathbf{r}^{\prime}$ at the upper end,

(3) $\mathbf{F}$ or $\mathbf{r}$ at $s_{\mathbf{1}}=\mathbf{0}$,

(4) $\mathbf{F}$ or $\mathbf{r}$ at the upper end, and

(5) the torsional moment at the lower or upper end.

The following points should be emphasized regarding the above model because they affect the numerical solution scheme significantly.

(1) Equilibrium of forces at the top of the riser yields (see Fig. 2):

$$
\begin{gathered}
F_{x, u}=T T F(\hat{t} \cdot \hat{i})-K_{x} x_{1, u} \\
F_{y, u}=\operatorname{TTF}(\hat{t} \cdot \hat{j})-K_{y} x_{2, u} \\
F_{z, u}=T T F(\hat{t} \cdot \hat{k})+T T R
\end{gathered}
$$

where

$$
T T F=\rho_{w} g \frac{\pi D_{0, u}^{2}}{4}\left[H_{w}-z_{u}\right]-\rho_{m} g \frac{\pi D_{i, u}^{2}}{4}\left[H_{m}-z_{u}\right]
$$

the subscript $u$ indicates the upper end of the riser, TTR is the tension applied at the top of the riser by the tensioning system and $K_{x}, K_{y}$ are spring constants. Equations (19)(22) show that these boundary forces are followers in nature (non-conservative), because the fluidic tension at the top of the riser given by equation (22) always acts in the tangential direction. ${ }^{18}$ Both TTF and its direction of application are deformation dependent. Further, by integrating equation (1) we get:

$$
\mathbf{F}\left(s_{1}\right)=\mathbf{F}\left(L_{1}\right)-\int_{s_{1}}^{L_{1}} \mathbf{q} \mathrm{d} s_{1}
$$

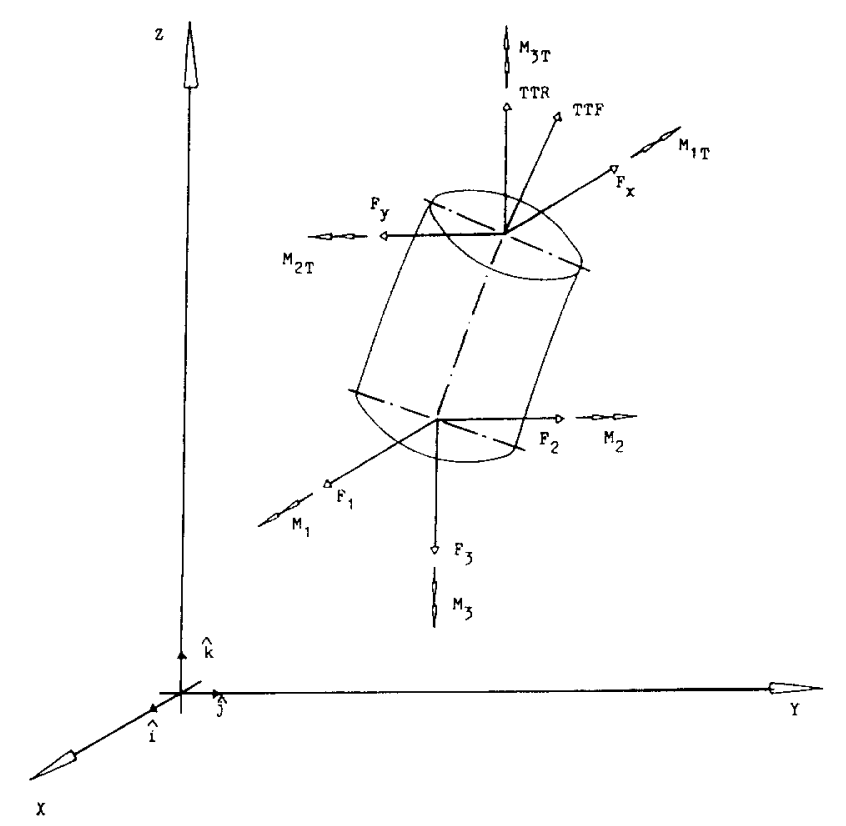

Figure 2. Differential element at the upper end. Internal and external forces 
where $L_{1}$ is the length of the deformed riser. Equation (23) shows that the internal force $F$, used to compute $T_{e}$ in (9), is deformation dependent. The nonlinearity of these boundary conditions and the deformation dependency are lost in linear models since the three directional cosines in (19), (20) and (21) become 0,0 and 1 respectively.

A similar set of conditions can be derived for the lower riser end. These complete boundary conditions can model cases of disconnected or connected risers, with any support rigidity.

(2) The effective tension $T_{e}$ needed in equation (4) can be computed in two ways. First by using equations (9) and (23) and second by using equations (6) and (10). This appears to be an inconsistency of the model. Actually it is not and simply implies that the first method should be used to evaluate $T_{e}$ and the second to compute $\epsilon_{t}$ for extensible risers. For inextensible risers, equation (24) must be used instead of (10):

$$
\epsilon_{t}=0
$$

(3) In static analysis the torsional moment $H$ is coupled with bending since in general $\mathbf{m}$ in equation (5) is nonzero. In recent publications on riser response $H$ is neglected. ${ }^{10,14,17,20,22,23}$ In this analysis $\mathrm{m}$ and $H$ are not neglected because they may be significant in some applications as stated in the rules for design and construction of risers developed by Det Norske Veritas. ${ }^{?}$

(4) Finally, it should be emphasized that in this work $T\left(s_{1}\right)$ is not considered as unknown as in refs. 10 and 20 because it can be expressed in terms of the degrees of freedom using equations (9) and (19)-(23).

\section{SOLUTION METHOD}

A numerical method for solving the mathematical model described by equations (1)-(23) is developed in this section. This method is based on an incremental finite element technique with a predictor-corrector scheme in each increment which is used to update the stiffness matrices, the boundary terms, the extensibility condition, and the deformation dependent loads (see Fig. 3).

The weak form of governing equation (4) can be derived by applying sequentially a Galerkin decomposition method and the incremental operator $\Delta$ as shown in Appendix $A$. The resulting equations are:

$$
\begin{aligned}
& B \int_{0}^{l_{1}} \Delta \mathbf{r}^{\prime \prime} \cdot \overrightarrow{\mathbf{r}}^{\prime \prime} \mathrm{d} s_{1}+\left(T_{e}-B \kappa^{2}\right) \int_{0}^{l_{1}} \Delta \mathbf{r}^{\prime} \cdot \overline{\mathbf{r}}^{\prime} \mathrm{d} s_{1} \\
& +\int_{0}^{l_{1}}\left(\mathbf{F} \cdot \Delta \mathbf{r}^{\prime}\right)\left(\mathbf{r}^{\prime} \cdot \overline{\mathbf{r}}^{\prime}\right) \mathrm{d} s_{1}-2 B \int_{0}^{l_{1}}\left(\mathbf{r}^{\prime} \cdot \Delta \mathbf{r}^{\prime \prime}\right)\left(\mathbf{r}^{\prime \prime} \cdot \overline{\mathbf{r}}^{\prime}\right) \mathrm{d} s_{1} \\
& +H \int_{0}^{l_{1}}\left(\Delta \mathbf{r}^{\prime} \times \mathbf{r}^{\prime \prime}\right) \cdot \overline{\mathbf{r}}^{\prime} \mathrm{d} s_{1}+H \int_{0}^{l_{1}}\left(\mathbf{r}^{\prime} \times \Delta \mathbf{r}^{\prime \prime}\right) \cdot \overline{\mathbf{r}}^{\prime} \mathrm{d} s_{1} \\
& +\int_{0}^{l_{1}}\left(\Delta \mathbf{r}^{\prime} \times \mathbf{m}\right) \cdot \overline{\mathbf{r}}^{\prime} \mathrm{d} s_{1}
\end{aligned}
$$

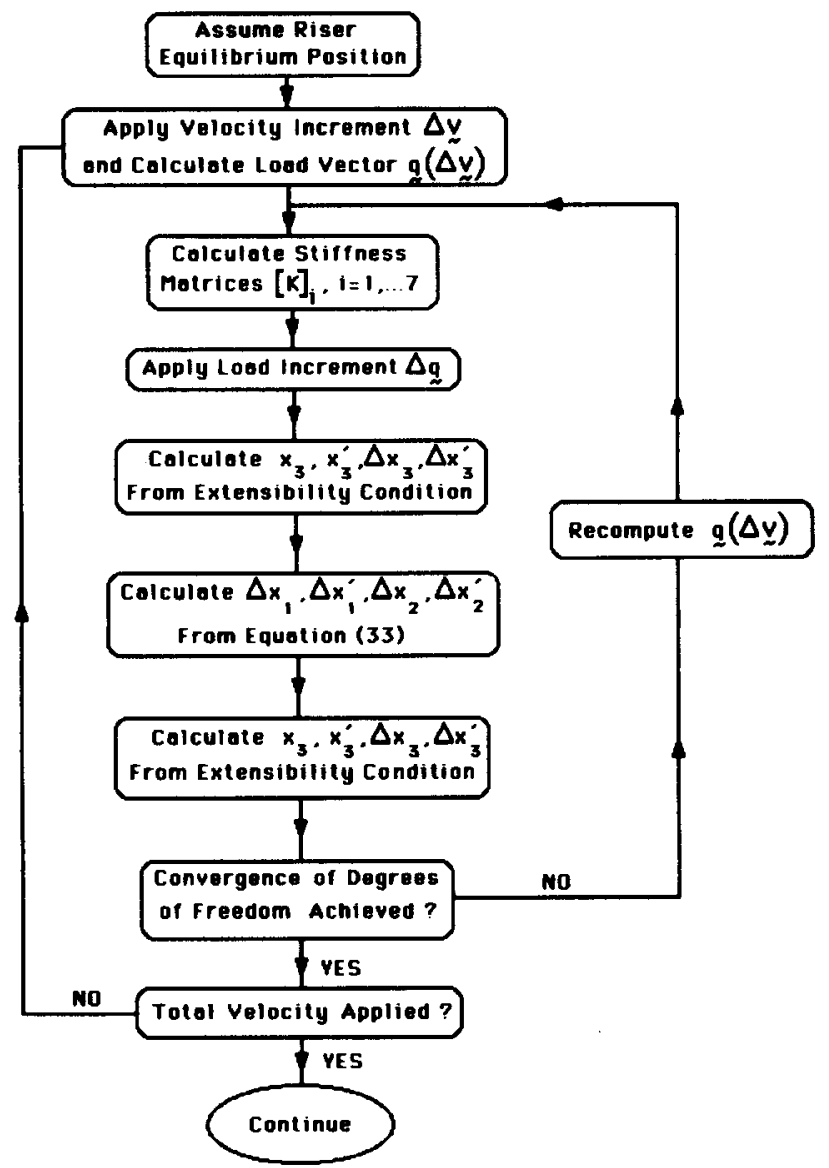

Figure 3. Solution method for the static large deformation analysis of riser

$$
\begin{aligned}
& =\int_{0}^{l_{1}} \Delta \mathbf{q} \cdot \overline{\mathbf{r}} \mathrm{d} s_{1}-\int_{0}^{l_{1}}\left(\Delta \mathbf{F} \cdot \mathbf{r}^{\prime}\right)\left(\mathbf{r}^{\prime} \cdot \overline{\mathbf{r}}^{\prime}\right) \mathrm{d} s_{1} \\
& -\Delta H \int_{0}^{l_{1}}\left(\mathbf{r}^{\prime} \times \mathbf{r}^{\prime \prime}\right) \cdot \overline{\mathbf{r}}^{\prime} \mathrm{d} s_{1}-\int_{0}^{l_{1}}\left(\mathbf{r}^{\prime} \times \Delta \mathbf{m}\right) \cdot \overline{\mathbf{r}}^{\prime} \mathrm{d} s_{1}+\left.\Delta \mathbf{F} \cdot \mathbf{\mathbf { r }}\right|_{0} ^{l_{1}} \\
& +\left.B \Delta \mathbf{r}^{\prime \prime} \cdot \overline{\mathbf{r}}^{\prime}\right|_{0} ^{l_{1}} \\
& \Delta H\left(s_{1}\right)=\Delta H(0)-\int_{0}^{s_{1}}(\Delta \hat{t} \cdot \mathbf{m}+\hat{t} \cdot \Delta \mathbf{m}) \mathrm{d} s_{1}
\end{aligned}
$$

and

$$
\Delta \mathrm{F}=\Delta \mathrm{F}\left(L_{1}\right)-\int_{s_{1}}^{L_{1}} \Delta \mathrm{q} \mathrm{d} s_{1}
$$

where equations (28) and (29) have been used:

$$
\begin{gathered}
\Delta T=\Delta \mathbf{F} \cdot \mathbf{r}^{\prime}+\mathbf{F} \cdot \Delta \mathbf{r}^{\prime} \\
\Delta \kappa^{2}=2 \Delta \mathbf{r}^{\prime \prime} \cdot \mathbf{r}^{\prime \prime}
\end{gathered}
$$

$\overline{\mathbf{r}}$ is the virtual displacement vector,

$$
l_{1}=\int_{0}^{l}\left(1+\epsilon_{t}\right) \mathrm{d} s
$$


and $l$ is the finite element length. It should be noted that in (25) the quantities in front of the integrals were considered constants for each element and were taken out of the integ. rals.

From the above weak form, the matrix equation (33) can be derived by using (31) and (32):

$$
\begin{array}{rlrl}
\Delta x_{j} & =\Delta x_{j \beta} N_{\beta} & \alpha, \beta & =1, \ldots, 4 \\
\bar{x}_{i} & =\bar{x}_{i \alpha} N_{\alpha} & i, j=1,2,3
\end{array}
$$

where $N_{\alpha}, N_{\beta}$ are the usual cubic beam shape functions and $\Delta x_{j \beta}$ are the 12 degrees of freedom of the element (six for each node):

$$
\left[\sum_{i=1}^{7}[\mathbf{K}]_{i}\right]\{\Delta \mathbf{x}\}=\{\mathbf{F}\}+\{\mathbf{G}\}
$$

The $i$ th stiffness matrix of (33) is derived from the $i$ th term on the left-hand side of equation $(25)$. $[\mathbf{K}]_{1}$ and $[\mathbf{K}]_{2}$ are symmetric, do not couple the riser response in directions $\hat{i}, \hat{j}$ and $\hat{k}$ and for an inextensible linear beam bending problem they reduce to the regular bending and geometric stiffness matrices. $[\mathbf{K}]_{3}$ and $[\mathbf{K}]_{4}$ are non-symmetric, full matrices. $[\mathbf{K}]_{5}$ and $[\mathbf{K}]_{7}$ are due to the torsional moment and distributed couple and are antisymmetric. They couple bending and torsion and are different from their counterparts in reference 17 where bending and torsion are uncoupled. $[\mathbf{K}]_{6}$ is a symmetric matrix.

$\{\mathbf{F}\}$ is the equivalent nodal force and moment vector defined by the first four integrals on the right-hand side of (25). $\{\mathbf{G}\}$ is the vector of internal forces and moments. The global equilibrium equation in matrix form is obtained by assembling the element equation (33) for all the riser elements.

The mathematical model described above has been implemented numerically and a computer code, STARI-3D, has been developed based on the following algorithm. The velocity is applied incrementally and the corresponding load increment is computed. For each load increment an iterative solution is employed with predictor and corrector phases in each iteration. During the prediction phase $x_{3}, x_{3}^{\prime}$, $\Delta x_{3}, \Delta x_{3}^{\prime}$, are computed using the kinematic relations (34)-(37):

$$
\begin{gathered}
x_{3}=x_{3}(0)+\int_{0}^{s_{1}} x_{3}^{\prime} \mathrm{d} s_{1} \\
x_{3}^{\prime}=\left(1-x_{2}^{\prime 2}-x_{1}^{\prime 2}\right)^{1 / 2}-\frac{1}{1+\epsilon_{t}} \\
\Delta x_{3}^{\prime}=\left[1+\frac{F_{3}}{E A\left(1+\epsilon_{t}\right)^{2}}\right]\left\{-\frac{x_{2}^{\prime} \Delta x_{2}^{\prime}+x_{1}^{\prime} \Delta x_{1}^{\prime}}{x_{3}^{\prime}+\left(1 /\left(1+\epsilon_{t}\right)\right)}\right. \\
+\frac{1}{E A\left(1+\epsilon_{t}\right)^{2}+F_{3}}\left[\Delta F_{1} x_{1}^{\prime}+\Delta F_{2} x_{2}^{\prime} \mathrm{d} s_{1}\right. \\
+\Delta F_{3}\left(x_{3}^{\prime}+\frac{1}{1+\epsilon_{t}}\right)+F_{1} \Delta x_{1}^{\prime}+F_{2} \Delta x_{2}^{\prime} \\
\left.\left.-\Delta x_{3} g\left(\rho_{m} A A_{i}-\rho_{w} A\right)\right]\right\}
\end{gathered}
$$

where equation (37) has been derived by elimination of $\Delta \epsilon_{t}$ from the incremental counterparts of equations (6), (9) and (35) as shown in Appendix B. In addition during the prediction phase $x_{1}^{\prime}, x_{1}^{\prime}, \Delta x_{1}, \Delta x_{1}^{\prime}, x_{2}, x_{2}^{\prime}, \Delta x_{2}, \Delta x_{2}^{\prime}$ are computed using (33). During the correction phase $x_{3}, x_{3}^{\prime}$, $\Delta x_{3}, \Delta x_{3}^{\prime}$ are recomputed using (34)-(37) and all stiffness matrices, the equivalent nodal forces, the boundary conditions and the lengths of the elements are corrected. Further $x_{1}, x_{1}^{\prime}, \Delta x_{1}, \Delta x_{1}^{\prime}, x_{2}, x_{2}^{\prime}, \Delta x_{2}, \Delta x_{2}^{\prime}$ are recomputed using (33). This prediction-correction scheme is repeated until convergence is achieved for each load increment. The process is repeated until the entire load is applied. The algorithm is summarized in Fig. 3.

\section{TEST CASES}

In order to test the accuracy of the computer program which was developed to implement the solution method described in the previous sections three test cases were ran. The first case is that of a cantilevered beam under uniform load. This problem has been solved in the past by several investigators using different semianalytical and numerical methods. Their results are compared to those of our computer code in Fig. 4. Holden ${ }^{13}$ used a Runge-Kutta method to integrate the nonlinear differential equation. Wang et $a l .^{27}$ used a finite difference method. Yang ${ }^{29}$ developed a finite element solution. Their results are in very good agreement with those derived in this work. Rhode's ${ }^{25}$ series solution underestimates by $5 \%$ the beam response. Wang's ${ }^{28}$ se second solution is in error as indicated by Holden ${ }^{13}$ and Reddy $^{24}$ because he commutes $\mathrm{d} / \mathrm{d} s$ and $\mathrm{d} / \mathrm{d} x$. Reddy develops and solves six different models, three of which overestimate and three underestimate the actual solution.

The second case is that of a simply supported beam under uniform load. Holden, ${ }^{13}$ Reddy $^{24}$ and Wang ${ }^{28}$ developed similar solutions like those described above for the cantilever beam (see Fig. 5). Iyengar ${ }^{16}$ using Rhode's ${ }^{25}$ method developed a series solution. Holden's and Iyengar's results compare very well to those derived in this work.

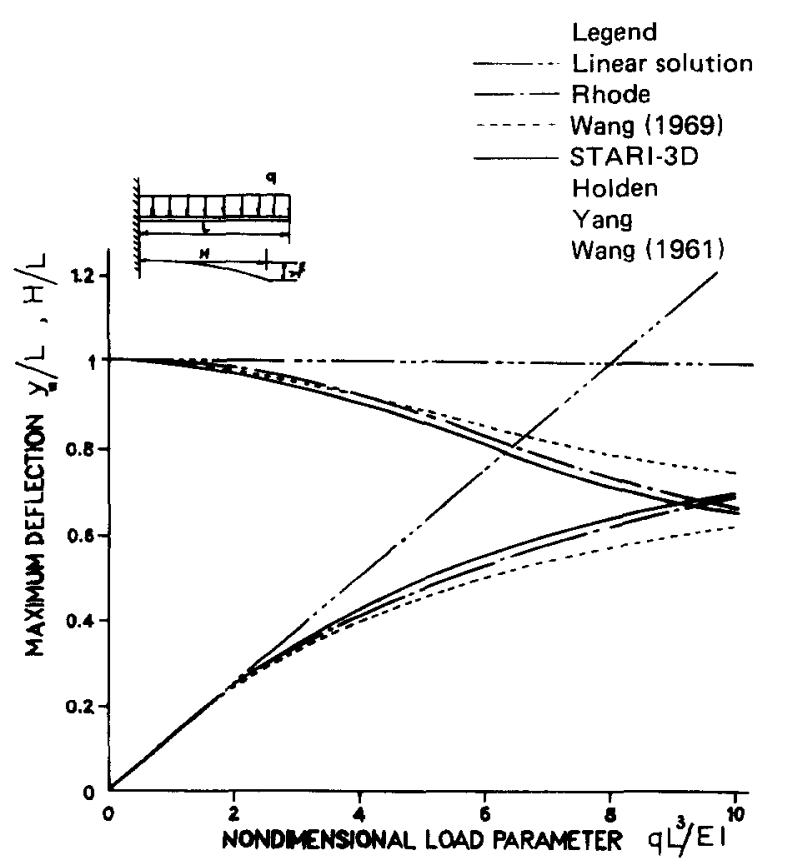

Figure 4. Comparison between large and small deformation models for a cantilever beam 


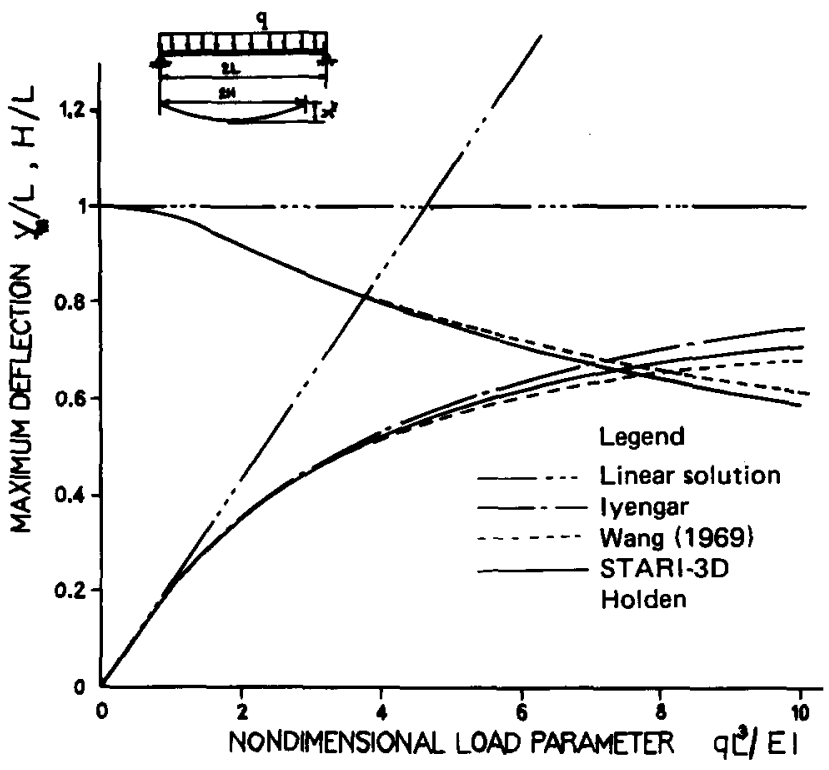

Figure 5. Comparison between large and small deformation models for a simply-supported beam

Table 1. Properties of riser used in the numerical applications

\begin{tabular}{lll}
\hline \multicolumn{1}{c}{ Property } & Symbol & \multicolumn{1}{c}{ Value } \\
\hline Length & $L$ & $2000 \mathrm{~m}$ \\
External diameter & $D_{o}$ & $0.610 \mathrm{~m}$ \\
Internal diameter & $D_{i}$ & $0.575 \mathrm{~m}$ \\
Buoyancy module diameter & $D_{b}$ & $0.870 \mathrm{~m}$ \\
Hydrodynamic diameter & $D_{H}$ & $0.870 \mathrm{~m}$ \\
Effective weight & $W_{e}$ & $1.1320 \mathrm{KN} / \mathrm{m}$ \\
Top tension & $T T R$ & $1.2 W_{e} L$ \\
Density of steel pipes & $\rho_{s t}$ & $8200 \mathrm{~kg} / \mathrm{m}^{3}$ \\
Density of water & $\rho_{w}$ & $1025 \mathrm{~kg} / \mathrm{m}^{3}$ \\
Density of buoyancy modules material & $\rho_{b m}$ & $440 \mathrm{~kg} / \mathrm{m}^{3}$ \\
Density of drilling internal fluid & $\rho_{m}$ & $1250 \mathrm{~kg} / \mathrm{m}^{3}$ \\
Offset of riser upper end & $\Delta$ & $0.0 \mathrm{~m}$ \\
Hydrodynamic coefficient & $C_{d}$ & $1.0 \mathrm{or} 0.7$ \\
\hline
\end{tabular}

Reddy's ${ }^{24}$ and Wang's ${ }^{28}$ solutions have the same problems like those described above for the solution they developed for the cantilever beam.

In the third test the riser was placed horizontally, a uniform two-dimensional vertical load was applied, the riser's bending and torsional rigidities were set equal to zero, one end was hinged and the other was placed on a horizontal roller with a known horizontal force applied to it in the vertical plane of the load. The results matched within an accuracy of $0.03 \%$ the catenary for a uniform cable.

To the best of our knowledge there are no test cases available in the literature for a three-dimensional marine riser problem. Thus further testing of the program is not currently possible. In the following section three numerical applications are presented which show the importance of three-dimensional nonlinear analysis.

\section{NUMERICAL APPLICATIONS}

The properties of the riser used in the numerical applications are summarized in Table 1.

Three different applications of a riser under static load are studied in this section in order to investigate the importance of structural nonlinearities and three-dimensional effects. In all applications the riser is hinged at both ends and 80 finite elements were used.

In the first application the riser is subject to a wind generated current with triangular velocity profile acting in the $x$ direction. The velocity of the current at the surface is $1.5 \mathrm{~m} / \mathrm{s}$ and drops linearly to zero at the sea bed. ${ }^{11}$ The drag coefficient $C_{d}$ is set equal to 1 . The riser response is analyzed using a linear program, and two two-dimensional versions of STARI-3D the first neglecting and the second taking into account the dependence of the external loads on the riser deformation. The analysis of risers should be done using the second version of STARI-3D. The other two programs are run in order to demonstrate the differences between the method developed in this work and those often used in analyzing the riser response by neglecting structural nonlinearities and/or the deformation dependency of the external loads. The results are summarized in Table 2 for the deflection and slope and in Figs. 6 and 7 for the bending and the Von Mises equivalent stress respectively. These stresses represent the maximum bending and equivalent stresses in any riser cross-section. The equivalent stress, $\sigma_{e}$, at any point is given by the Von Mises formula:

$$
\begin{aligned}
2 \sigma_{e}^{2}= & \left(\sigma_{x}-\sigma_{y}\right)^{2}+\left(\sigma_{y}-\sigma_{z}\right)^{2}+\left(\sigma_{z}-\sigma_{x}\right)^{2} \\
& +6\left(\tau_{x y}^{2}+\tau_{y z}^{2}+\tau_{z x}^{2}\right)
\end{aligned}
$$

where $\sigma_{x}, \sigma_{y}, \sigma_{z}$ are the normal and $\tau_{x y}, \tau_{y z}, \tau_{x z}$ the shear stresses in a local orthonormal system of co-ordinates. We can draw the following conclusions by studying these results. The differences in deflection between the linear and the first version of STARI-3D are small, of the order of $1 \%$, while those between the linear and the second version of

Table 2. Displacement and slope comparison between two-dimensional linear, nonlinear and nonlinear with deformation dependent load

\begin{tabular}{|c|c|c|c|c|c|c|}
\hline \multirow[b]{2}{*}{$z / L$} & \multirow[b]{2}{*}{ Linear } & \multicolumn{2}{|c|}{ Deflection (m) } & \multicolumn{3}{|c|}{ Slope (rad) } \\
\hline & & $\begin{array}{l}\text { Nonlinear } \\
\text { without } \\
\text { deformation } \\
\text { dependency }\end{array}$ & $\begin{array}{c}\text { Nonlinear } \\
\text { with } \\
\text { deformation } \\
\text { dependency }\end{array}$ & Linear & $\begin{array}{c}\text { Nonlinear } \\
\text { without } \\
\text { deformation } \\
\text { dependency }\end{array}$ & $\begin{array}{l}\text { Nonlinear } \\
\text { with } \\
\text { deformation } \\
\text { dependency }\end{array}$ \\
\hline 0.000 & 0.00 & 0.00 & 0.00 & 0.1979 & 0.1939 & 0.1759 \\
\hline 0.125 & 40.45 & 40.04 & 36.89 & 0.1275 & 0.1270 & 0.1194 \\
\hline 0.250 & 66.46 & 66.01 & 61.63 & 0.0832 & 0.0832 & 0.0803 \\
\hline 0.375 & 82.62 & 82.21 & 77.45 & 0.0464 & 0.0465 & 0.0462 \\
\hline 0.500 & 89.51 & 89.11 & 84.53 & 0.0081 & 0.0081 & 0.0097 \\
\hline 0.625 & 86.28 & 85.84 & 81.88 & -0.0350 & -0.0352 & -0.0320 \\
\hline $0.750^{\circ}$ & 71.47 & 70.97 & 67.99 & -0.0846 & -0.0848 & -0.0802 \\
\hline 0.875 & 43.35 & 42.88 & 41.22 & -0.1417 & -0.1410 & -0.1350 \\
\hline 1.000 & 0.00 & 0.00 & 0.00 & -0.2038 & -0.2010 & -0.1937 \\
\hline
\end{tabular}
analyses for risers subject to linear current 


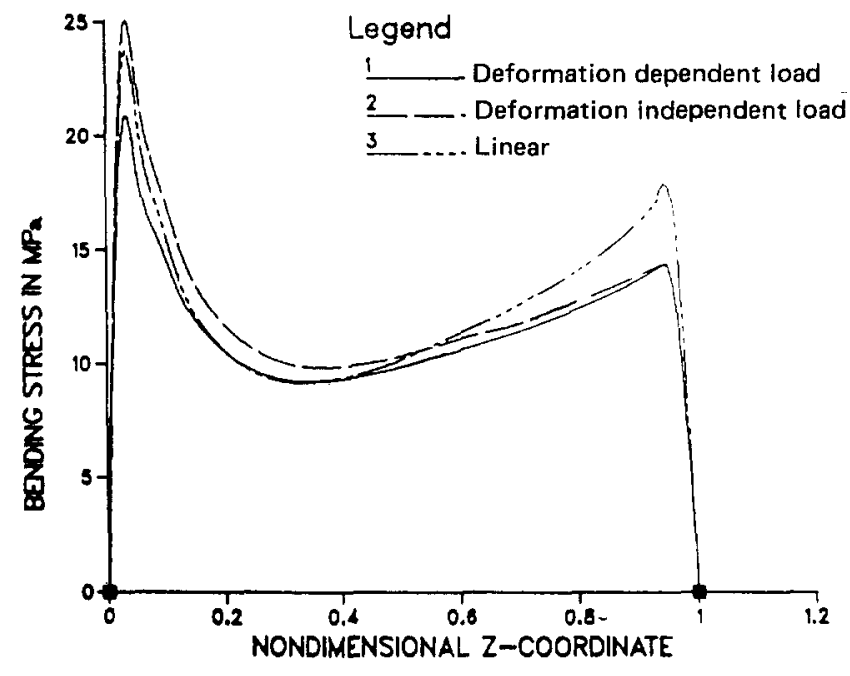

Figure 6. Bending stress for linear and nonlinear twodimensional analyses with or without deformation dependent load, for a riser subject to a linear current

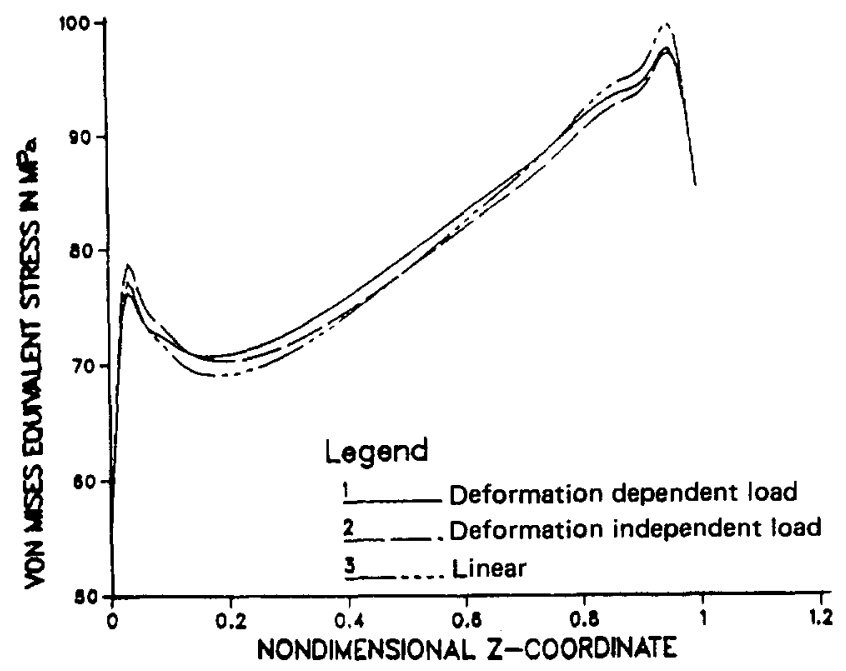

Figure 7. Von Mises equivalent stress for linear and nonlinear two-dimensional analyses with or without deformation dependent load, for a riser subject to a linear current

STARI-3D may be as much as $10 \%$. The differences in slope are up to $2 \%$ and $12.5 \%$ in these two cases respectively. The maximum differences in this example occur near the lower end of the riser because the internal tangential force is smaller than that near the upper end. The difference of $12.5 \%$ in the slope at the lower ball joint is very significant for operation of drilling risers and proper function of the ball joint. The linear model overestimates the bending stress by as much as $13.5 \%$ at the bottom, and $25 \%$ at the top with respect to the second version of STARI-3D. Further we note that in this application the linear program underestimates the bending stress near the lower ball joint and overestimates it near the upper end, compared to the first version of STARI-3D. Finally the differences between the three programs in the Von Mises equivalent stress are smaller, because this stress is dominated by the tensile component which does not vary significantly among these three cases.

In the second application the riser is subject to a tidal current with an exponential velocity profile acting in the $y$ direction. The velocity of the current at the surface is $1 \mathrm{~m} / \mathrm{s}$ and is assumed to decay proportionally to the seventh root of the depth to zero at the sea-bed. ${ }^{11}$ The drag coefficient $C_{d}$ is set equal to 1 . The riser response is analyzed again using the three programs described above. The results are presented in Table 3 for the deflection and slope in Figs. 8 and 9 for the bending and the Von Mises equivalent stress respectively. Similar conclusions to those derived in the previous application can be drawn. The difference between the correct analysis done by the second version of STARI$3 \mathrm{D}$ and the other two programs are more pronounced in this application. The slope at the lower ball joint is overestimated by the linear program by $17 \%$. Similarly, the bending stress is overestimated by up to $23 \%$ and the equivalent stress by up to $9 \%$ by the linear model.

In the third application the riser is subject to the tidal current described in the second application and applied in the $x$ direction, and to the linear current described in the first application and applied in the $y$ direction. The drag coefficient $C_{d}$ is set equal to 0.7 . The riser response is threedimensional and the results for the deflection and the bending, tensile and Von Mises equivalent stresses are summarized in Table 4 and Fig. 10 respectively. In Table 4 five different cases are considered in order to investigate the importance of three-dimensional analysis with deformation dependent loads. The correct results are those in the fifth and tenth column to which all others should be compared. The four cases compared to the correct one are:

(1) Two-dimensional nonlinear analysis with deformation independent loads in the $x$ and $y$ directions.

(2) Three-dimensional nonlinear analysis with deformation independent loads.

Table 3. Displacement comparison between two-dimensional linear, nonlinear and nonlinear with deformation dependent load analyses, for a riser subject to a tidal current

\begin{tabular}{|c|c|c|c|c|c|c|}
\hline \multirow[b]{2}{*}{$z / L$} & \multicolumn{3}{|c|}{ Deflection (m) } & \multicolumn{3}{|c|}{ Slope (rad) } \\
\hline & Linear & $\begin{array}{c}\text { Nonlinear } \\
\text { without } \\
\text { deformation } \\
\text { dependency }\end{array}$ & $\begin{array}{l}\text { Nonlinear } \\
\text { with } \\
\text { deformation } \\
\text { dependency }\end{array}$ & Linear & $\begin{array}{c}\text { Nonlinear } \\
\text { without } \\
\text { deformation } \\
\text { dependency }\end{array}$ & $\begin{array}{l}\text { Nonlinear } \\
\text { with } \\
\text { deformation } \\
\text { dependency }\end{array}$ \\
\hline 0.000 & 0.00 & 0.00 & 0.00 & 0.4204 & 0.3890 & 0.3603 \\
\hline 0.125 & 78.91 & 75.71 & 70.74 & 0.2130 & 0.2122 & 0.2014 \\
\hline 0.250 & 114.97 & 112.20 & 105.90 & 0.0868 & 0.0895 & 0.0881 \\
\hline 0.375 & 126.10 & 124.00 & 117.90 & 0.0075 & 0.0095 & 0.0121 \\
\hline 0.500 & 120.62 & 118.90 & 113.70 & -0.0485 & -0.0470 & -0.0428 \\
\hline 0.625 & 102.98 & 101.60 & 97.54 & -0.0909 & -0.0896 & -0.0846 \\
\hline 0.750 & 75.90 & 74.83 & 72.10 & -0.1246 & -0.1231 & -0.1178 \\
\hline 0.875 & 41.15 & 40.53 & 39.16 & -0.1525 & -0.1504 & -0.1449 \\
\hline 1.000 & 0.00 & 0.00 & 0.00 & -0.1753 & -0.1728 & -0.1672 \\
\hline
\end{tabular}


Table 4. Deflection comparison, between two- and three-dimensional nonlinear analyses, with or without deformation dependent load, for a riser subject to a tidal and $a$ wind current

\begin{tabular}{|c|c|c|c|c|c|c|c|c|c|c|}
\hline \multirow[b]{3}{*}{$z / L$} & \multicolumn{5}{|c|}{ Deflection, $x_{1}(\mathrm{~m})$} & \multicolumn{5}{|c|}{ Deflection. $x_{2}(\mathrm{~m})$} \\
\hline & \multicolumn{2}{|c|}{$\begin{array}{c}\text { Without deformation } \\
\text { dependency }\end{array}$} & \multicolumn{3}{|c|}{ With deformation dependency } & \multicolumn{2}{|c|}{$\begin{array}{l}\text { Without deformation } \\
\text { dependency }\end{array}$} & \multicolumn{3}{|c|}{ With deformation dependency } \\
\hline & 2-D & 3-D & 2-D & 3-D* & $\begin{array}{c}\text { 3-D w/o } \\
\text { vertical } \\
\text { hydro- } \\
\text { dynamic } \\
\text { load }\end{array}$ & 2-D & $3-D$ & 2-D & $3-D^{*}$ & $\begin{array}{c}\text { 3-D w/o } \\
\text { vertical } \\
\text { hydro- } \\
\text { dynamic } \\
\text { load }\end{array}$ \\
\hline 0.000 & 0.00 & 0.00 & 0.00 & 0.00 & 0.00 & 0.00 & 0.00 & 0.00 & 0.00 & 0.00 \\
\hline 0.125 & 45.49 & 43.94 & 42.19 & 38.65 & 41.64 & 65.33 & 64.20 & 60.89 & 56.65 & 61.84 \\
\hline 0.250 & 73.36 & 71.74 & 68.86 & 64.13 & 68.37 & 98.45 & 97.19 & 92.76 & 87.32 & 94.12 \\
\hline 0.375 & 88.78 & 87.30 & 84.03 & 79.07 & 83.52 & 111.20 & 110.00 & 105.60 & 100.20 & 106.80 \\
\hline 0.500 & 93.35 & 92.01 & 88.91 & 84.19 & 88.15 & 109.40 & 108.20 & 104.50 & 99.60 & 105.10 \\
\hline 0.625 & 87.27 & 86.05 & 83.55 & 79.41 & 82.39 & 95.93 & 94.86 & 92.07 & 88.01 & 91.93 \\
\hline 0.750 & 70.15 & 69.11 & 67.45 & 64.23 & 65.98 & 72.58 & 71.62 & 69.93 & 66.90 & 69.13 \\
\hline 0.875 & 41.31 & 40.63 & 39.86 & 37.98 & 38.60 & 40.37 & 39.70 & 39.03 & 37.30 & 38.10 \\
\hline 1.000 & 0.00 & 0.00 & 0.00 & 0.00 & 0.00 & 0.00 & 0.00 & 0.00 & 0.00 & 0.00 \\
\hline
\end{tabular}

*All cases should be compared to this one

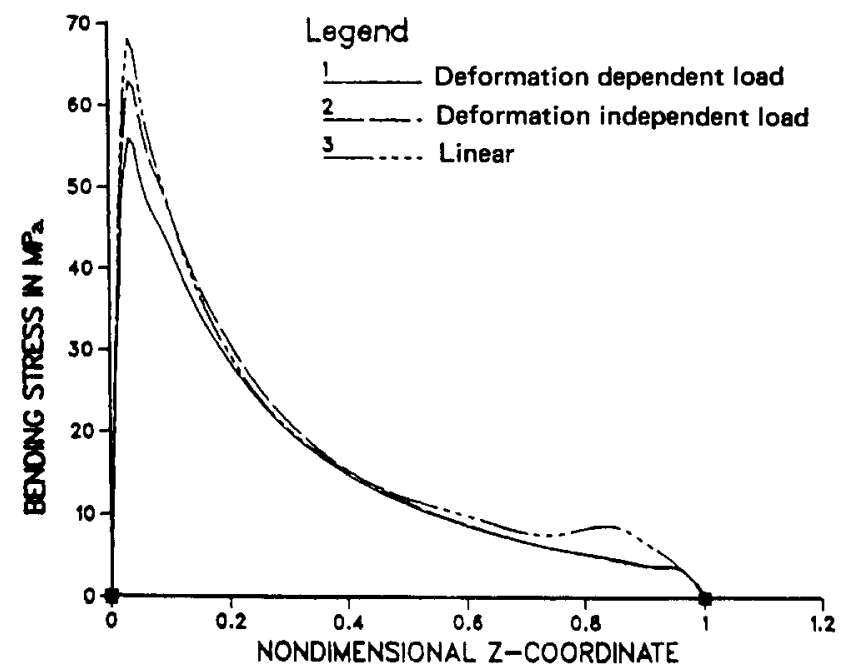

Figure 8. Bending stress for linear and nonlinear two. dimensional analyses with or without deformation dependent load, for a riser subject to a tidal current

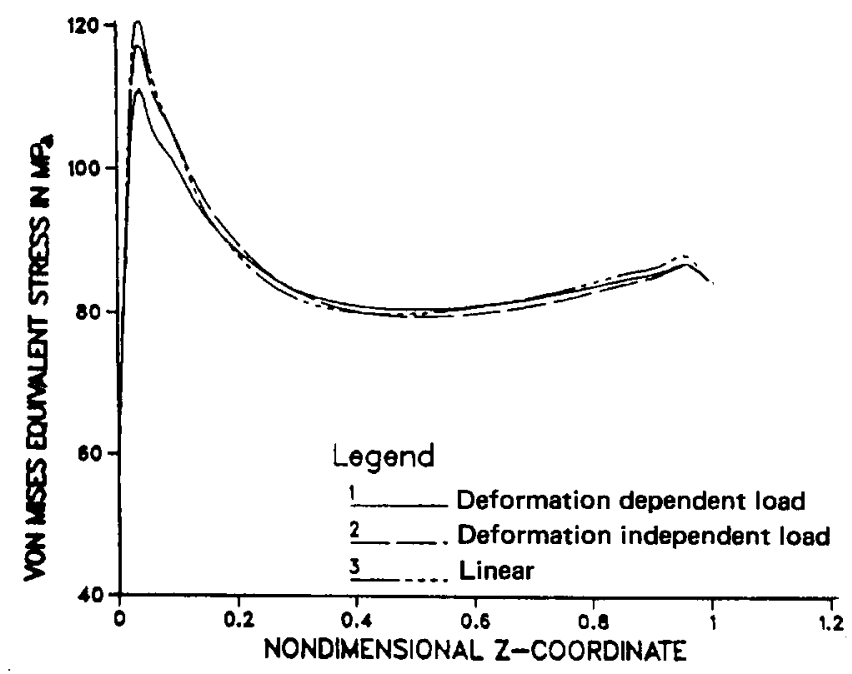

Figure 9. Von Mises equivalent stress for linear and nonlinear two-dimensional analyses with or without deformation dependent load, for a riser subject to a tidal current

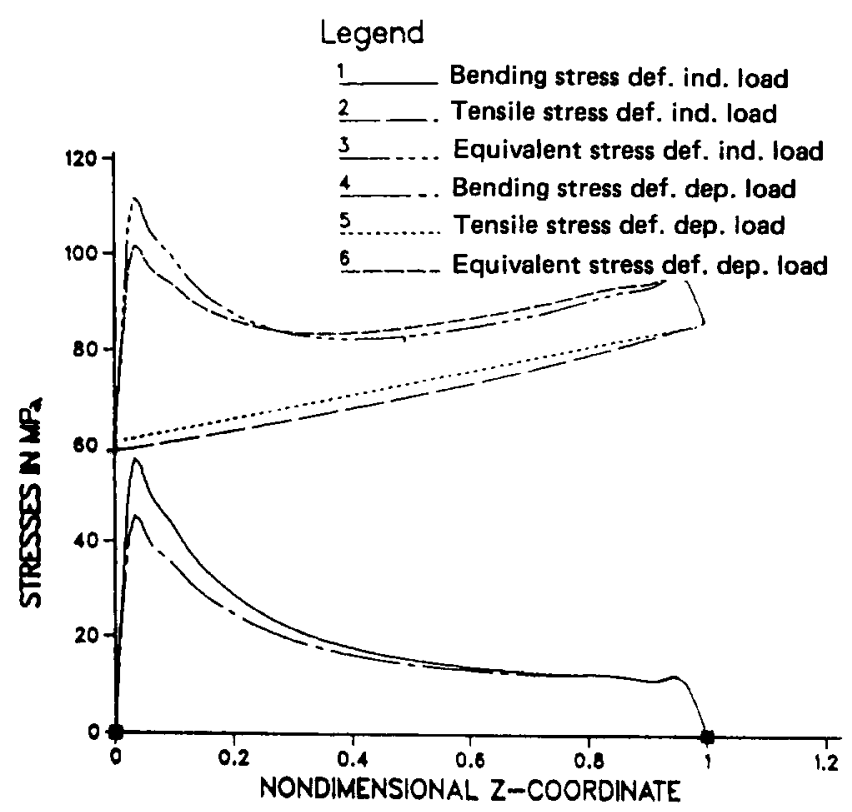

Figure 10. Stresses for three-dimensional nonlinear analyses for a riser subject to a tidal and a wind current

(3) Two-dimensional nonlinear analysis with deformation dependent loads in the $x$ and $y$ directions.

(4) Three-dimensional nonlinear analysis with deformation dependent loads, ignoring the hydrodynamic load in the vertical direction.

We can draw the following conclusions. Three-dimensional coupling is more important in deformation dependent than in deformation independent loading. Further, it has a strong stiffening effect which reduces the deformation by as much as $11.5 \%$. The differences in the five cases are more pronounced near the lower ball joint where the tangential force is relatively small. Finally, the vertical hydrodynamic load component should not be neglected as is often done in riser analysis.

Regarding the bending, tensile and equivalent stresses in the riser we compare the three-dimensional analyses with and without deformation dependent loading. The results 
depicted in Fig. 10 show that the dependency of loads on deformation must not be neglected, since that would result in overestimation of bending stresses by up to $26.5 \%$ and equivalent stresses by up to $9 \%$. The tensile stress, on the contrary, is slightly underestimated because in deformation independent loading, the hydrodynamic load component in the $z$ direction is neglected.

\section{CONCLUSIONS}

A comprehensive three-dimensional nonlinear large deformation model has been developed for marine risers and solved using an incremental finite element method involving an iterative scheme with predictor and corrector phases in each increment. The algorithm can handle the deformation dependent boundary conditions and loads, the extensibility or inextensibility condition and torsion. Three test cases have shown the high accuracy of the developed computer code. Three numerical applications have shown that the stiffening effects of nonlinearities and the deformation dependency of the loads are important both for two- and three-dimensional riser analyses. They may result in drastic reduction of deflection, slopes, bending stresses and equivalent stresses particularly near the lower ball joint. These differences are very pronounced in comparisons of the analysis developed in this work to the traditional analyses using structurally linear and hydrodynamically nonlinear models.

\section{ACKNOWLEGEMENTS}

Part of this work has been supported by the Ocean Engineering Division of the American Bureau of Shipping.

\section{REFERENCES}

1 Bennett, B. E. and Metcalf, M. F. 'Nonlinear dynamic analysis of coupled axial and lateral motions of marine risers', O.T.C. Paper No. 2776, 1977

2 Borgman, L. E. "Computation of the ocean-wave forces on inclined cylinders', American Geophysical Union, Transactions 1958, 39 (5)

3 Bernitsas, M. M. 'Three-dimensional nonlinear large deflection model for dynamic behavior of risers, pipelines and cables', Journal of Ship Research 1982, 26 (1)

4 Bernitsas, M. M. and Guha-Thakurta, S. 'HYDCYL: a database for calculation of HYDrodynamic Loading of Circular CYLinders', Report No. 267, Department of Naval Architecture and Marine Engineering, The University of Michigan, 1984

5 Chakrabarti, S. K., Tam, W. A. and Wolbert, A. L. 'Wave forces on a randomly oriented tube', Proc. of Offshore Technology Conf., Paper No. 2190, 1975, pp. 433-47

6 Chakrabarti, S. K. and Frampton, R. E. 'Review of riser analysis techniques', Applied Ocean Research 1982, 4 (2)

7 Det Norske Veritas, 'Rules for the design, construction and inspection of submarine pipelines and pipeline risers', Hovik, Norway, 1976

8 Eisenhart, L. P. An Introduction to Differential Geometry, Princeton University Press, Princeton, 1947

9 Felippa, C. A. and Chung, J. S. 'Nonlinear static analysis of deep ocean mining pipe', Journal of Energy Resources Technology 1981, 107

10 Garrett, D. L. 'Dynamic analysis of slender rods', Journal of Energy Resources Technology 1982, 104

11 Gaythwaite, T. The Marine Environment and Structural Design, Van Nostrand Reinhold Co., 1981

12 Gnone, E., Signorelli, P. and Giuliano, V. 'Three-dimensional static and dynamic analysis of deep water sealines and risers', O.T.C. Paper 2326, 1975

13 Holden, J. T. 'On the finite deflection of thin beams', Int. Journal of Solids and Structures 1972, 8, 1051

14 Huang, T. and Chucheepsakul, S. 'Large displacement analysis of a marine riser', Proc. 3rd Offshore and Arctic Engineering Symp., New Orleans, 1984
15 Imron, A. 'Nonlinear static three-dimensional analysis of risers", P.E. Dissertation, Dept of Naval Architecture and Marine Engineering, The University of Michigan, 1984

16 Iyengar, K. T. and Rao, S. K. 'Large deflections of simply supported beams', Journal of Franklin Institute, 1955, 259, 523

17 Kim, Y. C. and Triantafyllou, M. S. 'The nonlinear dynamics of long, slender cylinders', Proc. 3rd Offshore and Arctic Engineer. ing Symp., New Orleans, 1984

18 Kokkinis, T. and Bernitsas, M. M. 'Postbuckling analysis of heavy columns with application to marine risers', J. Ship Research (in press)

19 Konuk, I. 'Application of an adaptive numerical technique to 3-D pipeline problems with strong nonlinearities', Journal Energy Resources Technology 1982, 104

20 Larsen, C. M. and Kavlie, D. Nonlinear Analysis of Oil Pipelines by Potential Minimization, Division of Ship Structures, The Norwegian Institute of Technology, 1977

21 Love, A. E. H. A Treatise on the Mathematical Theory of Elasticity, Dover, New, York, 1927

22 Nordgren, R. P. 'On computation of the motion of elastic rods, Journal Applied Mech. 1974, 96, 777

23 Nordyren, R. P. 'Dynamic analysis of marine risers with vortex excitation', Journal Energy Resources Technology 1982, 104

24 Reddy, J. N. and Singh, I. R. 'Large deflections and large amplitude free vibrations of straight and curved beams', Int. Journal for Numerical Methods in Engineering 1981, 17, 829

25 Rhode, F. V. 'Large deflections of cantilever beams with uniformly distributed load', Quarterly of Applied Mathematics $1953,11,337$

26 Safai, H. V. 'Nonlinear dynamics analysis of deep water risers', Applied Ocean Research 1983, 5 (4)

27 Wang, T. M., Lee, S. L. and Zienkiewicz, O. C. 'A numerical analysis of large deflection of beams', Int. Journal of Mechani cal Science 1961, 219

28 Wang, T. M. 'Non-linear bending of beams with uniformly distributed loads', Int. Journal of Non-linear Mechanics 1969 , 4, 389

29 Yang, T. Y. 'Matrix displacement solution to elastis problems of beams and frames', Int. Journal of Solids and Structures $1973,3,829$

\section{APPENDIX A}

\section{WEAK FORM OF GOVERNING EQUATION (4)}

Multiplying equation (4) by the virtual displacement vector $\overline{\mathbf{r}}$, and integrating over the element length $l_{1}$ given by equation (30), we get:

$$
\begin{aligned}
& \int_{0}^{l_{1}}\left\{\left(B \mathbf{r}^{\prime \prime}\right)^{\prime \prime} \cdot \overline{\mathbf{r}}-\left[\left(T_{e}-B \kappa^{2}\right) \mathbf{r}^{\prime}\right]^{\prime} \cdot \overline{\mathbf{r}}-\left[H\left(\mathbf{r}^{\prime} \times \mathbf{r}^{\prime \prime}\right)\right]^{\prime} \cdot \overline{\mathbf{r}}\right. \\
& \left.-\left(\mathbf{r}^{\prime} \times \mathbf{m}\right)^{\prime} \cdot \overline{\mathbf{r}}\right\} \mathrm{d} s \\
& =\int_{0}^{l_{1}} \mathbf{q} \cdot \overline{\mathbf{r}} \mathrm{d} s
\end{aligned}
$$

Integrating (A1) by parts we obtain:

$$
\begin{aligned}
& \int_{0}^{l_{1}} B \mathbf{r}^{\prime \prime} \cdot \overline{\mathbf{r}}^{\prime \prime} \mathrm{d} s+\int_{0}^{l_{1}}\left[\left(T_{e}-B \kappa^{2}\right) \mathbf{r}^{\prime}\right] \cdot \overline{\mathbf{r}}^{\prime} \mathrm{d} s+\int_{0}^{l_{1}} H\left(\mathbf{r}^{\prime} \times \mathbf{r}^{\prime \prime}\right) \cdot \overline{\mathbf{r}}^{\prime} \mathrm{d} s \\
& \quad+\int_{0}^{l_{1}}\left(\mathbf{r}^{\prime} \times \mathbf{m}\right) \cdot \overline{\mathbf{r}}^{\prime} \mathrm{d} s \\
& =\int_{0}^{l_{1}} \mathbf{q} \cdot \overline{\mathbf{r}} \mathrm{d} s+\left.\mathbf{F}(s) \cdot \overline{\mathbf{r}}\right|_{0} ^{l_{1}}+\left.(\hat{t} \times \mathbf{M}(s)) \cdot \overline{\mathbf{r}}^{\prime}\right|_{0} ^{l_{1}}
\end{aligned}
$$


Further, by applying on both sides of equation (A2) the incremental operator $\Delta$ we get equation (25). $\Delta$ has the meaning of a difference operator. Specifically:

$$
\Delta x=x_{k+1}-x_{k}
$$

where $x$ is an independent variable and the subscript indicates the increment number. The operator applied to a function $h$ yields:

$$
\Delta h=h_{k+1}-h_{k}
$$

Equation (A4) can be further analyzed by using Taylor's expansion. For example, if $h$ is function of independent variables $x$ and $y, \Delta h$ can be written in the form:

$$
\Delta h \cong \frac{\partial h}{\partial x} \Delta x+\frac{\partial h}{\partial y} \Delta y+\mathrm{O}\left(\Delta x^{2}, \Delta y^{2}, \Delta x \Delta y\right)
$$

\section{APPENDIX B \\ INCREMENTAL FORM OF THE EXTENSIBILITY EQUATION}

The extensibility equation (35) can be written as:

$$
x_{1}^{\prime 2}+x_{2}^{\prime 2}+\left(x_{3}^{\prime}+\frac{1}{1+\epsilon_{t}}\right)^{2}=1
$$

Applying the incremental operator $\Delta$ on (B1) we get:

$$
\Delta x_{3}^{\prime}=-\frac{x_{2}^{\prime} \Delta x_{2}^{\prime}+x_{1}^{\prime} \Delta x_{1}^{\prime}}{x_{3}^{\prime}+\left(1 /\left(1+\epsilon_{t}\right)\right)}+\frac{\Delta \epsilon_{t}}{\left(1+\epsilon_{t}\right)^{2}}
$$

Further $\Delta \epsilon_{t}$ is computed by eliminating $\Delta T_{e}$ from equations (B3) and (B4):

$$
\begin{gathered}
\Delta T_{e}=\Delta F_{1} x_{1}^{\prime}+\Delta F_{2} x_{2}^{\prime}+\Delta F_{3}\left(x_{3}^{\prime}+\frac{1}{1+\epsilon_{t}}\right)+F_{1} \Delta x_{1}^{\prime} \\
+F_{2} \Delta x_{2}^{\prime}+F_{3} \Delta x_{3}^{\prime}-F_{3} \frac{\Delta \epsilon_{t}}{\left(1+\epsilon_{t}\right)^{2}} \\
\Delta T_{e}=E A \Delta \epsilon_{t}-\Delta x_{3} g\left(\rho_{w} A_{0}-\rho_{m} A_{i}\right)
\end{gathered}
$$

where (B3) and (B4) are the incremental forms of equations (9) and (6) respectively. Substituting the resulting expression for $\Delta \epsilon_{t}$ in equation (B2) and solving for $\Delta x_{3}^{\prime}$, we obtain equation (37). 